\title{
Activation of GPR30 improves exercise capacity and skeletal muscle strength in senescent female Fischer344×Brown Norway rats
}

\author{
Hao Wang ${ }^{\mathrm{a}, \mathrm{b}}$, Allan Alencar ${ }^{\mathrm{a}, \mathrm{e}}$, Marina Lin ${ }^{\mathrm{a}}$, Xuming Sun ${ }^{\mathrm{a}}$, Roberto T. Sudo ${ }^{\mathrm{a}, \mathrm{e}}$, Gisele \\ Zapata-Sudo $^{\mathrm{a}, e}$, Dawn A. Lowe ${ }^{f}$, and Leanne Groban ${ }^{\mathrm{a}, \mathrm{b}, \mathrm{c}, \mathrm{d}}$ \\ Allan Alencar: allankdc@gmail.com; Marina Lin: mlin@wakehealth.edu; Xuming Sun: xsun@wakehealth.edu; Roberto T. \\ Sudo: rtsudo@icb.efrj.bf; Gisele Zapata-Sudo: gzsudo@oi.com.br; Dawn A. Lowe: lowex017@umn.edu; Leanne Groban: \\ Igroban@wakehealth.edu \\ aDepartment of Anesthesiology, Wake Forest School of Medicine, Medical Center Boulevard, \\ Winston-Salem, North Carolina, USA \\ bInternal Medicine/Molecular Medicine, Wake Forest School of Medicine, Medical Center \\ Boulevard, Winston-Salem, North Carolina, USA \\ cHypertension and Vascular Research Center, Wake Forest School of Medicine, Medical Center \\ Boulevard, Winston-Salem, North Carolina, USA \\ dSticht Center on Aging, Wake Forest School of Medicine, Medical Center Boulevard, Winston- \\ Salem, North Carolina, USA \\ eInstitute of Biomedical Sciences, Drug Development Program, Federal University of Rio de \\ Janeiro, Brazil \\ fPrograms in Rehabilitation Science and Physical Therapy, Dept. of Physical Medicine and \\ Rehabilitation, University of Minnesota, Minneapolis, Minnesota, USA
}

\section{Abstract}

The molecular mechanisms of muscle weakness and sarcopenia in postmenopausal women are largely unknown. To determine the effect of a new estrogen receptor, GPR30, in the maintenance of exercise capacity and skeletal muscle function in females, the selective GPR30 agonist, G1 (100 $\mu \mathrm{g} / \mathrm{kg} / \mathrm{day}$ ), or vehicle ( $\mathrm{V}$, soybean oil) was administered subcutaneously daily ( $\mathrm{n}=7$ per group) to ovariectomized (OVX) 27-month-old Fischer 344×Brown Norway (F344BN) female rats. Following 8 weeks of treatment, the exercise capacity (treadmill walk time to exhaustion) was reduced in OVX vs. sham rats $(5.1 \pm 1.4$ vs. $11.0 \pm 0.9 \mathrm{~min}, P<0.05)$, and chronic G1 restored exercise capacity $(12.9 \pm 1.2 \mathrm{~min} ; P<0.05 \mathrm{vs}$. OVX-V). Similarly, the peak twitch of electrically stimulated soleus muscles was decreased by $22 \%$ in OVX $v s$. sham rats $(P<0.05)$, and $\mathrm{G} 1$

Address correspondence to: Hao Wang, MD, PhD, Department of Anesthesiology, Wake Forest School of Medicine, Medical Center Boulevard, Winston-Salem, NC 27157-1009, Phone: 336-716-4498, Fax: 336-716-8190, ; Email: haowang @ wakehealth.edu Publisher's Disclaimer: This is a PDF file of an unedited manuscript that has been accepted for publication. As a service to our customers we are providing this early version of the manuscript. The manuscript will undergo copyediting, typesetting, and review of the resulting proof before it is published in its final citable form. Please note that during the production process errors may be discovered which could affect the content, and all legal disclaimers that apply to the journal pertain. 
attenuated this decline $(P<0.05)$. Western blot analysis showed that chronic G1 treatment attenuated OVX-associated decreases in heat shock protein (HSP) 90, HSP70, and HSP27 expressions. In vitro studies using the L6 myoblast cell line demonstrated that G1 increased mRNA levels of HSPs in cultured cells. Collectively, these data demonstrate that the activation of GPR30 mitigates the adverse effects of estrogen loss on exercise capacity and skeletal muscle contractile function in old F344BN rats. The protective effects of GPR30 might be through its upregulation of heat shock proteins in skeletal muscle.

\section{Keywords}

GPR30; exercise capacity; skeletal muscle; ovariectomy; Fischer344×Brown Norway rat

\section{Introduction}

The Western world is getting older and the number of older adults (individuals $>65$ years) in our society is expected to almost triple by 2050 . One of the most common health problems in older people is a decline in physical function and disability. Although numerous factors contribute to the declines in physical function, the loss of muscle mass (sarcopenia), strength, and power appear to be the main reason for the physical dysfunction and disability. We lack proven pharmacologic therapies to delay or reverse age-related muscle weakness and sarcopenia since the molecular mechanisms of this deterioration are still largely unknown [1]. Investigating the mechanisms underlying this condition and developing efficient interventions for the prevention and treatment of muscle abnormalities during aging would be of great importance for health care professionals.

The research on skeletal muscle aging in women is even more important since, compared to age-matched males, older women tend to have more severe sarcopenia, less muscle strength and power, and a greater risk for disability [2]. Moreover, since women tend to live longer than men, a greater number of older women are affected by physical disabilities. Sarcopenia and skeletal muscle weakness in older women accelerate after menopause, whereas in men of the same age, age-related declines in muscle structure and function are more gradual [3]. A correlation between muscle strength and circulating estrogen levels has also been reported [4]. These findings suggest a link between sarcopenia, skeletal muscle weakness, and estrogen loss. Although animal studies show that estradiol (E2) replacement improves skeletal muscle function in ovariectomized (OVX) mice and rats [5,6], clinical trials of estrogen-based hormone therapy in postmenopausal women have been contradictory with respect to physical function [7]. Moreover, given the possible increased risk of cardiovascular disease and breast cancer associated with estrogen-based hormone therapy [8,9], further investigations on the molecular mechanisms that underlie sarcopenia and skeletal muscle weakness in aged females after the loss of ovarian function are critical in order to develop more specific treatments with lower side effects.

GPR30 is a newly recognized estrogen receptor expressed in various tissues, including skeletal muscle. Moreover, in female C57BL/6 mice, GPR30 expression is several fold greater in skeletal muscle and brain compared to liver, lung, kidney, heart, and fat $[10,11]$. 
However, its functions in skeletal muscle and its responsiveness to changes in circulating estrogen are unclear. Given that GPR30 activation protects cardiac muscle from stresses including estrogen loss in animal models [12,13], we hypothesize that activation of GPR30 by its selective agonist G1 has protective effects in skeletal muscle of old Fischer $344 \times$ Brown Norway (F344BN) rats that were assured ovarian hormone deficient by ovariectomy.

\section{Methods}

Animals

Twenty-six-month-old female F344BN rats were purchased from the National Institute on Aging colony at Harlan Industries (Indianapolis, IN, USA) and were studied after 1 month of acclimation to their housing conditions. Rats were housed two per cage and were maintained on a 12-hour light/dark cycle, at constant temperature and humidity, with ad libitum access to standard rat chow and tap water. All studies were approved by the Institutional Animal Care and Use Committee (IACUC) at Wake Forest School of Medicine (Approved protocol \#A12-201).

\section{Experimental Protocol}

At 27 months of age, rats underwent either bilateral OVX or a sham procedure as described previously [13]. Once the rats reached 28 months of age, the OVX group was randomized to receive either G1 (100 $\mu$ g/kg/day; Cayman Chemical Company, Ann Arbor, MI) or vehicle (V; soybean oil; Xcelience Inc., Tampa, FL) by daily subcutaneous administration for 8 weeks. Sham-operated rats were injected with vehicle as a control. Treadmill tests were performed 1 day before surgery and at 35, 60, and 90 days after surgery. At 30 months of age, rats were euthanized via exsanguination by cardiac puncture (ketamine $\mathrm{HCl} 60 \mathrm{mg} / \mathrm{kg}$ and xylazine HCL $5 \mathrm{mg} / \mathrm{kg}$ ). Hind leg skeletal muscles were dissected for ex vivo functional studies or stored in $-80^{\circ} \mathrm{C}$.

\section{Exercise Capacity Test}

The maximal exercise capacity test (time to exhaustion during a standardized exercise protocol) was performed using a motorized-treadmill. Rats were familiarized with the motor-driven, one-lane rodent treadmill (Scientific Instruments, Stoelting, Wood Dale, IL) by walking at a speed of $20 \mathrm{~cm} / \mathrm{s}, 10 \mathrm{~min} / \mathrm{d}$, for 1 week. Each exercise test was performed after at least 1 day of rest. The protocol for exercise capacity evaluation consisted of $3 \mathrm{~min}$ at 12 meters/min, with 1.2-meter/min increases in speed every $3 \mathrm{~min}$ until the rats reached exhaustion. Time to exhaustion (in minutes) was determined when the rats stopped running and sat at the lower end of the treadmill for more than 5 seconds, despite gentle nudging by an investigator (ML) who was blinded to the study groups.

\section{Muscle Collection}

Skeletal muscles from bilateral hind limbs were dissected, and soleus (SOL), extensor digitorum longus (EDL), and gastrocnemius (GAS) muscles were isolated and weighed. The soleus muscles from the right side were immediately used for determination of contractile function and fatigue. The soleus from the left side and all bilateral EDL and GAS muscles 
were immediately frozen in liquid nitrogen and stored at $-80^{\circ} \mathrm{C}$ for subsequent $\mathrm{PCR}$ and western blot analyses.

\section{Contraction and Fatigue Study in Soleus Muscle}

Isolated soleus muscles were tied with silk suture at the myotendinous junctions and then hung vertically between a bottom fixed hook and a top isometric force transducer (Grass force transducer FT03, Astro Med, Inc., West Warwick, RI), which was connected to a micromanipulator. The muscles were positioned in an organ bath chamber filled with KrebsRinger solution oxygenated with $95 \% \mathrm{O}_{2} / 5 \% \mathrm{CO}_{2}$ maintained at $\mathrm{pH} 7.4$ and $37^{\circ} \mathrm{C}$. Muscles were then allowed to rest for $30 \mathrm{~min}$ before starting the contractile protocol. The electrical current delivered by a pair of platinum electrodes connected to a voltage controlled stimulator (Grass S48, Astro Med, Inc. West Warwick, RI) generated an electrical field to induce muscle twitches. Force data were collected (Axoscope 10.2, Molecular Devices, Union City, California), digitized and stored in a personal computer for further analysis. For these baseline twitch recordings, the rate and pulse duration of stimulation were set to 0.2 $\mathrm{Hz}$ and $2 \mathrm{~ms}$, respectively.

For the fatigue study, twitch forces at baseline $\left(\mathrm{Pt}_{0}\right)$ and at the end of 3 minutes of stimulation $\left(\mathrm{Pt}_{3}\right)$ at the rate of $3 \mathrm{~Hz}$ were analyzed. The fatigue index $(\mathrm{FI})$ was calculated as $\mathrm{FI}=\mathrm{Pt}_{3} / \mathrm{Pt}_{0}$.

\section{Western blot Analysis}

Muscle homogenates were separated by SDS-PAGE and transferred onto membranes, as previously described [13]. Immunoblots were probed by antibodies against GPR30 (1:500, Bioss, Woburn, MA, USA), heat shock protein (HSP) 90 (1:1000, BD Biosciences, San Jose CA, USA), HSP70 (1:1000, BD Biosciences), HSP27 (1:1000, Abcam, Inc., Cambridge, MA, USA), and Glyceraldehyde-3-phosphate dehydrogenase (GAPDH; 1:5,000; Cell Signaling, Danvers, MA, USA) was used as a loading control.

\section{Quantitative Real-time RT-PCR (qPCR)}

qPCR was used to detect gene mRNA levels in EDL and GAS muscles using a SYBR Green PCR kit (Qiagen Inc., Valencia, California, USA), as we previously described [13]. Amplification and detection were performed with the ABI7500 Sequence Detection System (Applied Biosystems, Foster City, CA). The relative target mRNA levels in each sample were normalized to GAPDH RNA. Expression levels are reported relative to the geometric mean of the control group.

\section{Cell Culture and Treatment}

The L6 myoblast cells (ATCC; Manassas, VA) were grown in Phenol Red-free Dulbecco's modified Eagle's medium (DMEM, Gibco) with $10 \%$ fetal bovine serum (FBS), at $37^{\circ} \mathrm{C}$ in $5 \% \mathrm{CO}_{2}$ and $95 \%$ air, at a relative humidity of $95 \%$. Before the experiment, cells were seeded in six-well plates at the density of $5 \times 10^{4}$ cells $/ \mathrm{cm}^{2}$ and starved for $18 \mathrm{~h}$ in DMEM without FBS. Cells were subsequently treated with G1 $\left(10^{-6}-10^{-9} \mathrm{M}\right)$ or Vehicle. After 1 hour incubation, cells were lysed with TRIzol (Invitrogen, Carlsbad, CA, USA) for real-time RT-PCR analysis for HSP mRNA levels. 


\section{Statistical Analysis}

All results are reported as mean \pm SEM. For all endpoints, one-way ANOVA was used to determine the significance of differences among groups. Significant interactions between groups were determined using Tukey post-hoc tests. Differences for all tests were considered significant at $P<0.05$. Analyses were performed using GraphPad Prism, version 6 (GraphPad, San Diego, CA, USA).

\section{Results \\ GPR30 Expression in Skeletal Muscles}

To study the direct effects of GPR30 activation on rat skeletal muscle, we first determined the expression of GPR30 in skeletal muscles. Results from RT-PCR show that the GPR30 gene exists in soleus muscle of old female F344BN rats (Fig. 1A). Using a specific antibody to GPR30, we found a single band in Western blot analysis corresponding to the appropriate molecular weight of GPR30 ( $\sim 50 \mathrm{kDa}$ ) in SOL (Fig. 1B). Furthermore, in OVX vs. shamoperated rats, real-time PCR showed that GPR30 mRNA increased by 1.2-fold in EDL $(P<0.05)$, and tended to increase in GAS muscles; these changes were inhibited by chronic G1 treatment (Fig. 1C).

\section{G1 Attenuates the Adverse Effects of Estrogen Loss on Exercise Capacity}

The maximal exercise capacity (minutes to exhaustion), determined by the graded treadmill test, is shown in Fig. 2A. The time to exhaustion in sham-operated rats remained unchanged at 35-, 63-, and 84-days post-surgery when compared to pre-surgical exercise times. In contrast, age-matched, OVX-V rats exhibited a significant reduction in exercise capacity at 35-days post-surgery compared to baseline, and this impairment in exercise persisted throughout the study. One week of G1 treatment did not alter the maximal exercise capacity in OVX-G1 rats when compared to their OVX-vehicle-treated counterparts. However, following a longer treatment of G1, OVX-related exercise intolerance recovered. Specifically, at 63- and 84-days post-surgery, OVX-G1 rats exhibited exercise tolerance times equivalent to their vehicle-treated, ovary-intact counterparts.

\section{Soleus Muscle Contractile Properties}

To evaluate the physiological effects of chronic G1 treatment versus vehicle in skeletal muscle from gonadal intact vs. OVX rats, muscle contractile characteristics from SOL muscles were measured. SOL muscle mass was not different among the three groups of rats $(0.225 \pm 0.008 \mathrm{~g}$ for all, $P>0.05)$. Estrogen loss by OVX reduced the peak twitch force by $26 \%$ compared with gonadal intact rats $(41.1 \pm 4.3 v s .56 .4 \pm 5.0 \mathrm{mN}, P<0.05)$, which was restored by treatment with $\mathrm{G} 1$ to $59.6 \pm 3.1 \mathrm{mN}$ (Fig. 2B).

The susceptibility to fatigue of the soleus was assessed by repetitive stimulation of the muscle ex vivo. A typical tracing is shown in the Fig. S1A, in which the amplitude of twitches decrease with the time of stimulation. Despite the difference in peak force generation, no difference in the rate of fatigue development existed among groups, as reflected by similar slopes of the decline in the twitch development curve. After $3 \mathrm{~min}$ of stimulation, twitch amplitude was reduced by $46 \%, 40 \%$, and $44 \%$ in sham, OVX-V, and 
OVX-G1 rats, respectively (Fig. S1B). There were no statistical differences among groups $(P>0.05)$.

\section{G1 Inhibits the Reduction of HSP90, HSP70, and HSP27 in Soleus Associated with Estrogen Loss}

In the soleus muscles, Western blot analysis showed the protein expressions of HSP90, HSP70, and HSP27 were dramatically decreased in OVX rats compared with sham operated rats. G1 treatment in OVX rats restored expression levels of these HSPs to that of their sham operated, gonadal-intact counterparts (Fig. 3).

\section{Mitochondrial and Apoptosis-related Gene Expression in EDL and GAS}

Induction of HSPs has been shown to improve mitochondrial function and protect cells against apoptosis. Therefore, the expression of transcription factor genes involved in the biogenesis of mitochondria and apoptosis-related genes were investigated. As shown in Fig. 4, estrogen loss by OVX induced BAX mRNA increases only in EDL and Bcl-2 mRNA decreases only in GAS. NRF-1, NRF-2b, and TFB2M mRNA levels increased in OVX rats in GAS, but not in EDL. OVX increased COX I mRNA in EDL but decreased gene expression in GAS. COX IV and TFAM mRNA decreased only in EDL of OVX rats. These gene expression changes, excluding COX IV and TFAM, were attenuated by G1 treatment (Fig. 4), suggesting that the protective effects of GPR30 in skeletal muscle might be via favorable changes in mitochondria and apoptosis-related genes.

\section{G1 Increases mRNA Levels of Heat Shock Proteins in L6 Skeletal Muscle Cells}

Consistent with results in rat muscle, real-time PCR showed that 1 hour of G1 treatment in cultured L6 skeletal muscle cells increased HSP mRNA expression. G1 increased HSP27 mRNA at a dose of $1 \mu \mathrm{M}$, increased HSP70 mRNA at a dose of 0.01-0.1 $\mu \mathrm{M}$, and increased HSP90 mRNA at a dose of 0.1-1 $\mu \mathrm{M}$ (Fig. S2).

\section{Discussion}

The most important findings of this study are: 1) Chronic GPR30 activation by its agonist G1 attenuated the adverse effects of late-life estrogen loss on excise capacity in the female F344BN rats, 2) G1 inhibited OVX-induced decreases in peak twitch and HSP90, HSP70, and HSP27 expression in the soleus muscle, and 3) G1 treatment increased mRNA levels of these HSPs in cultured L6 skeletal muscle cells. These results suggest that the GPR30 receptor is important in the maintenance of sex-specific skeletal muscle structure and function in aged females, probably through its regulation on HSPs expression in skeletal muscle.

Although clinical evidence suggest an association between estrogen loss and sarcopenia and skeletal muscle weakness in postmenopausal women [3,4], effects of estrogen, especially its individual receptors, on the structure and function of skeletal muscle are not clear. Studies using an OVX rat model with estrogen receptor (ER) subtype-selective agonists showed that the ER $\beta$ isoform protects skeletal muscle in response to high-intensity treadmill runninginduced injury or in nutrition-induced obesity [14,15]; ERa had beneficial effects on satellite 
cell activation after exercise-induced muscle injury $[14,16]$. However, the mechanisms of age and menopause-related sarcopenia and muscle strength loss are not understood. This study demonstrates that GPR30 activation by G1 attenuates reductions in treadmill exercise capacity and soleus muscle twitch force following ovariectomy in aged female F344BN rats, suggesting that GPR30 activation might mediate the protective effects of estrogen in skeletal muscle during aging and after estrogen loss.

It has been reported that GPR30 is highly expressed in skeletal muscles of both male and female mice $[10,11]$. This study demonstrates that GPR30 message and protein are also expressed in rat skeletal muscles. Moreover, GPR30 mRNA increases in EDL and tends to increase in GAS muscles of OVX rats compared to gonadal-intact rats. This is novel information showing first, that the receptor is expressed in aged muscle; and, second, that it is responsive to hormone status, likely reflecting a compensatory mechanism in response to estrogen loss in the ovariectomized rats, as we reported in cardiac muscle [13]. Given that GPR30 is expressed in various other tissues, subcutaneous administration of G1 could affect skeletal muscle indirectly through its systemic effects on the whole body, such as through metabolic changes [17]. However, considering the high level of GPR30 expression in skeletal muscle [10,11], together with our in vitro results showing G1 increased HSPs gene expression in cultured L6 skeletal muscle cells, and the protective effects of GPR30 in cardiac muscle [12,13], it is most likely that the beneficial effects of GPR30 activation by chronic G1 treatment in the aged female F344BN rat was at least partly through direct effects on skeletal muscle cells. Indeed, more specific and powerful approaches including the use of skeletal muscle-specific GPR30 knockout mice are needed to pinpoint the role of GPR30 in skeletal muscle and the underlying mechanisms.

The protective effects of G1 are associated with the upregulation of HSP90, HSP70, and HSP27 expressions in skeletal muscles of ovariectomized F344BN rats. HSPs are a highly conserved family of proteins that act as "molecular chaperones," and play important roles in maintaining cellular homeostasis in response to stress. Induction of HSPs has been shown to enhance metabolic characteristics, inhibit inflammatory pathways, decrease oxidative stress, improve mitochondrial function, and protect cells against apoptosis [18,19]. Different HSPs are expressed in skeletal muscle including HSP27, HSP70, and HSP90, which respond to a wide variety of pathological, environmental and physiological stresses including exercise and aging [20,21]. Given that lifelong elevations of HSP in skeletal muscle provides protection against damage and facilitates successful recovery after damage in muscles of old mice [22], the findings in the present study strongly suggest that the upregulation of HSPs might mediate the protective effects of G1 in skeletal muscles after estrogen loss. This concept is also supported by the favorable changes of mitochondrial and apoptosis-related gene expression after G1 treatment in OVX rats, which is consistent with previous findings showing that HSPs improve mitochondrial function [19], and protect cells against apoptosis [23]. Apoptosis of skeletal muscle fibers and satellite cells is considered a possible contributor to aging decrements that occur in skeletal muscle $[23,24]$.

How HSP expressions are regulated by sex hormones, especially in skeletal muscle, is not yet clear. Numerous preclinical studies show that females express higher endogenous levels of HSPs, which are decreased upon OVX, and E2 treatment leads to increased expression of 
HSPs in various tissues including skeletal muscle [25,26]. In vitro studies have demonstrated that E2 treatment significantly increased HSP27 and HSP72 expressions in C2C12 skeletal muscle cells, cardiomyocytes, and breast cancer cells [27,28]. In the present study, HSP90, HSP70, and HSP27 protein levels in the soleus dramatically decreased in OVX rats compared with sham rats, while G1 treatment restored these levels to that of their gonadalintact, sham-operated counterparts . Furthermore, our in vitro study showed that G1 treatment increased HSP90, HSP70, and HSP27 mRNA levels in cultured L6 skeletal muscle cells. These data provide direct evidence suggesting that the regulation of HSPs expression by estrogen, as previously reported [25-28], might be mediated by its receptor GPR30, which is highly expressed in skeletal muscle.

\section{Potential Clinical Value}

Determining the molecular mechanisms that underlie sarcopenia and skeletal muscle weakness in women after estrogen loss is critical to the development of more specific treatments with lower side effects. This study has demonstrated that the activation of a newly discovered estrogen receptor, GPR30, by its specific agonist, G1, attenuated OVX-associated decreases in exercise capacity, soleus twitch, and expression of HSPs in skeletal muscle. These findings have advanced our understanding of how estrogen protects the skeletal muscle, and provides the impetus for future clinical studies focusing on the efficacy of GPR30/HSP activation in the prevention and treatment of sarcopenia and skeletal muscle weakness in postmenopausal women.

\section{Supplementary Material}

Refer to Web version on PubMed Central for supplementary material.

\section{Acknowledgments}

This work was funded in part by National Institutes of Health grants AG-033727 to L.G., and AG031743 to D.L.

\section{References}

[1]. Nedergaard A, Henriksen K, Karsdal MA, Christiansen C. Musculoskeletal ageing and primary prevention. Best Pract. Res. Clin. Obstet. Gynaecol. 2013; 27:673-688. [PubMed: 23891483]

[2]. Bouchard DR, Héroux M, Janssen I. Association between muscle mass, leg strength, and fat mass with physical function in older adults: influence of age and sex. J. Aging Health. 2011; 23:313328. [PubMed: 21081704]

[3]. Maltais ML, Desroches J, Dionne IJ. Changes in muscle mass and strength after menopause. J. Musculoskelet. Neuronal. Interact. 2009; 9:186-197. [PubMed: 19949277]

[4]. Cauley JA, Gutai JP, Kuller LH, LeDonne D, Powell JG. The epidemiology of serum sex hormones in postmenopausal women. Am. J. Epidemiol. 1989; 129:1120-1131. [PubMed: 2729251]

[5]. Bunratsami S, Udomuksorn W, Kumarnsit E, Vongvatcharanon S, Vongvatcharanon U. Estrogen replacement improves skeletal muscle performance by increasing parvalbumin levels in ovariectomized rats. Acta. Histochem. 2015; 117:163-175. [PubMed: 25578914]

[6]. Moran AL, Nelson SA, Landisch RM, Warren GL, Lowe DA. Estradiol replacement reverses ovariectomy-induced muscle contractile and myosin dysfunction in mature female mice. J. Appl. Physiol. (1985). 2007; 102:1387-1393. [PubMed: 17218423] 
[7]. Greising SM, Baltgalvis KA, Lowe DA, Warren GL. Hormone therapy and skeletal muscle strength: a meta-analysis. J. Gerontol. A. Biol. Sci. Med. Sci. 2009; 64:1071-1081. [PubMed: 19561145]

[8]. Hale GE, Shufelt CL. Hormone therapy in menopause: An update on cardiovascular disease considerations. Trends. Cardiovasc. Med. 2015; 25:540-549. [PubMed: 26270318]

[9]. Hvidtfeldt UA, Tjønneland A, Keiding N, et al. Risk of breast cancer in relation to combined effects of hormone therapy, body mass index, and alcohol use, by hormone-receptor status. Epidemiology. 2015; 26:353-361. N. [PubMed: 25695354]

[10]. Mårtensson UE, Salehi SA, Windahl S, et al. Deletion of the G protein-coupled receptor 30 impairs glucose tolerance, reduces bone growth, increases blood pressure, and eliminates estradiol-stimulated insulin release in female mice. Endocrinology. 2009; 150:687-698. [PubMed: 18845638]

[11]. Baltgalvis KA, Greising SM, Warren GL, Lowe DA. Estrogen regulates estrogen receptors and antioxidant gene expression in mouse skeletal muscle. PLoS. One. 2010; 5:e10164. [PubMed: 20405008]

[12]. Zhao Z, Wang H, Jessup JA, Lindsey SH, Chappell MC, Groban L. Role of estrogen in diastolic dysfunction. Am. J. Physiol. Heart. Circ. Physiol. 2014; 306:H628-H640.

[13]. Wang H, Jessup JA, Lin MS, Chagas C, Lindsey SH, Groban L. Activation of GPR30 attenuates diastolic dysfunction and left ventricle remodelling in oophorectomized mRen2.Lewis rats. Cardiovasc. Res. 2012; 94:96-104. [PubMed: 22328091]

[14]. Velders M, Diel P. How sex hormones promote skeletal muscle regeneration. Sports. Med. 2013; 43:1089-100. [PubMed: 23888432]

[15]. Velders M, Schleipen B, Fritzemeier KH, Zierau O, Diel P. Selective estrogen receptor- $\beta$ activation stimulates skeletal muscle growth and regeneration. FASEB. J. 2012; 26:1909-1920. [PubMed: 22278942]

[16]. Perry MC, Dufour CR, Tam IS, B'chir W, Giguère V. Estrogen-related receptor-a coordinates transcriptional programs essential for exercise tolerance and muscle fitness. Mol. Endocrinol. 2014; 28:2060-2071. [PubMed: 25361393]

[17]. Sharma G, Prossnitz ER. GPER/GPR30 knockout mice: Effects of GPER on metabolism. Methods. Mol. Biol. 2016; 1366:489-502. [PubMed: 26585159]

[18]. Silverstein MG, Ordanes D, Wylie AT, et al. Inducing muscle heat shock protein 70 improves insulin sensitivity and muscular performance in aged mice. J. Gerontol. A. Biol. Sci. Med. Sci. 2015; 70:800-808. [PubMed: 25123646]

[19]. Henstridge DC, Bruce CR, Drew BG, et al. Activating HSP72 in rodent skeletal muscle increases mitochondrial number and oxidative capacity and decreases insulin resistance. Diabetes. 2014; 63:1881-1894. [PubMed: 24430435]

[20]. Kim JS, Lee YH, Choi DY, Yi HK. Expression of heat shock proteins (HSPs) in aged skeletal muscles depends on the frequency and duration of exercise training. J. Sports. Sci. Med. 2015; 14:347-353. [PubMed: 25983584]

[21]. Beltran Valls MR, Wilkinson DJ, Narici MV, et al. Protein carbonylation and heat shock proteins in human skeletal muscle: relationships to age and sarcopenia. J. Gerontol. A. Biol. Sci. Med. Sci. 2015; 70:174-181. [PubMed: 24621945]

[22]. McArdle A, Dillmann WH, Mestril R, Faulkner JA, Jackson MJ. Overexpression of HSP70 in mouse skeletal muscle protects against muscle damage and age-related muscle dysfunction. FASEB. J. 2004; 18:355-357. [PubMed: 14688209]

[23]. Rice KM, Manne ND, Gadde MK, Paturi S, Arvapalli R, Blough E. Differential regulation of apoptosis in slow and fast twitch muscles of aged female F344BN rats. Age. (Dordr). 2015; 37:30. [PubMed: 25813803]

[24]. Sciorati C, Rigamonti E, Manfredi AA, Rovere-Querini P. Cell death, clearance and immunity in the skeletal muscle. Cell. Death. Differ. 2016 In Press.

[25]. Bombardier E, Vigna C, Bloemberg D, Quadrilatero J, Tiidus PM, Tupling AR. The role of estrogen receptor-a in estrogen-mediated regulation of basal and exercise-induced Hsp70 and Hsp27 expression in rat soleus. Can. J. Physiol. Pharmacol. 2013; 91:823-829. [PubMed: 24303535] 
[26]. Romani WA, Russ DW. Acute effects of sex-specific sex hormones on heat shock proteins in fast muscle of male and female rats. Eur. J. Appl. Physiol. 2013; 113:2503-2510. [PubMed: 23821238]

[27]. Vasconsuelo A, Milanesi L, Boland R. Participation of HSP27 in the antiapoptotic action of 17beta-estradiol in skeletal muscle cells. Cell. Stress. Chaperones. 2010; 15:183-192. [PubMed: 19621276]

[28]. Stice JP, Chen L, Kim SC, et al. 17 $\beta$-Estradiol, aging, inflammation, and the stress response in the female heart. Endocrinology. 2011; 152:1589-1598. [PubMed: 21303943] 


\section{Highlights}

- GPR30 activation reverses adverse effects of OVX on exercise capacity in rats.

- GPR30 activation prevents OVX-related reductions in peak twitch in soleus muscle.

- GPR30 activation inhibits OVX-induced decrease in HSP expression in soleus muscle.

- GPR30 activation increases HSP expression in cultured L6 skeletal muscle cells. 

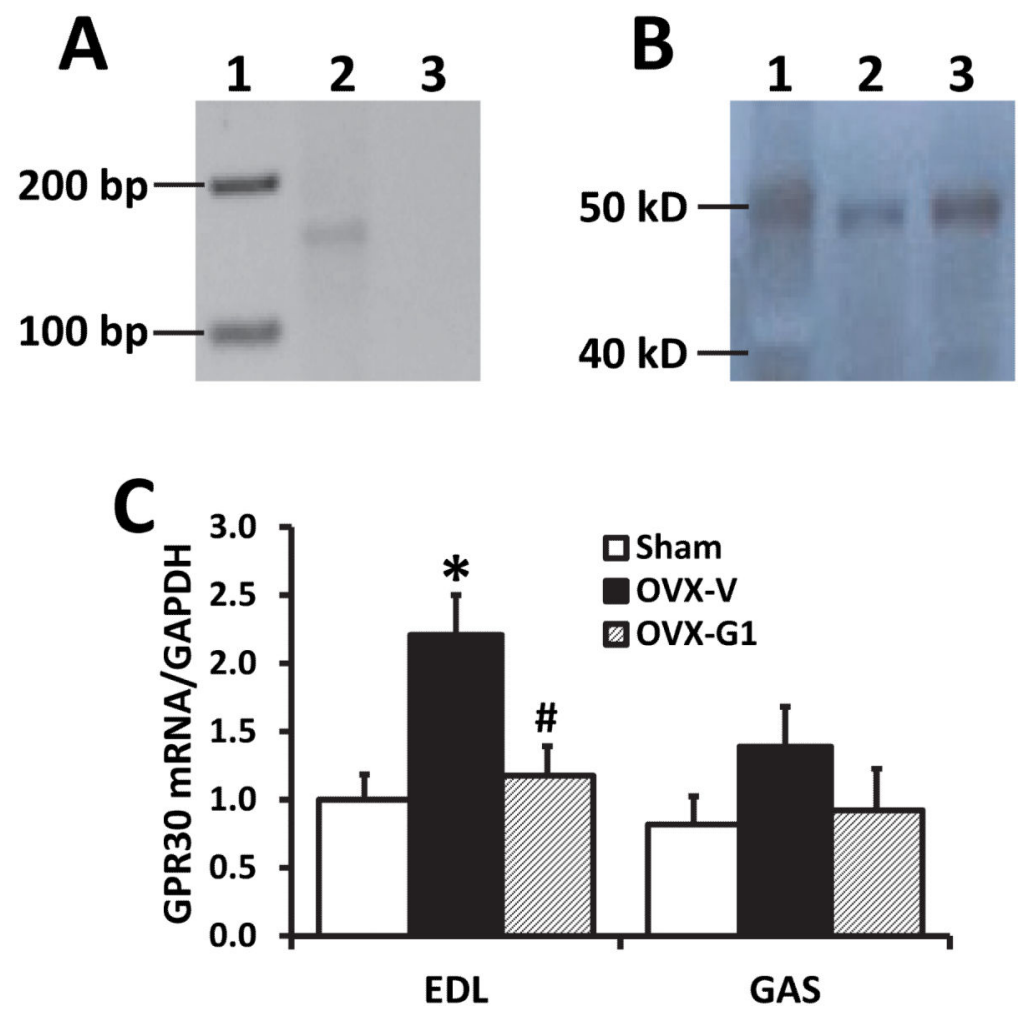

Fig. 1. GPR30 expression in skeletal muscles of female F344BN rats

(A) RT-PCR amplification of a 151-bp GPR30 fragment in soleus muscle. Lane 1, marker; lane 2, soleus muscle; lane 3, negative control (without cDNA). (B) Western blot of soleus muscle showing a single band corresponding to the appropriate molecular weight of GPR30 ( 50 kDa). Lane 1, marker; lane 2-3, soleus muscle samples. (C) GPR30 mRNA level, determined by real-time PCR, in extensor digitorum longus (EDL) and gastrocnemius (GAS) of sham-operated and ovariectomized (OVX) female F344BN rats treated with vehicle or G1 for 2 months. Values are mean \pm SEM; $* P<0.05$ vs. sham; \# $P<0.05$ vs. OVX-V. $\mathrm{n}=7$ /group. 

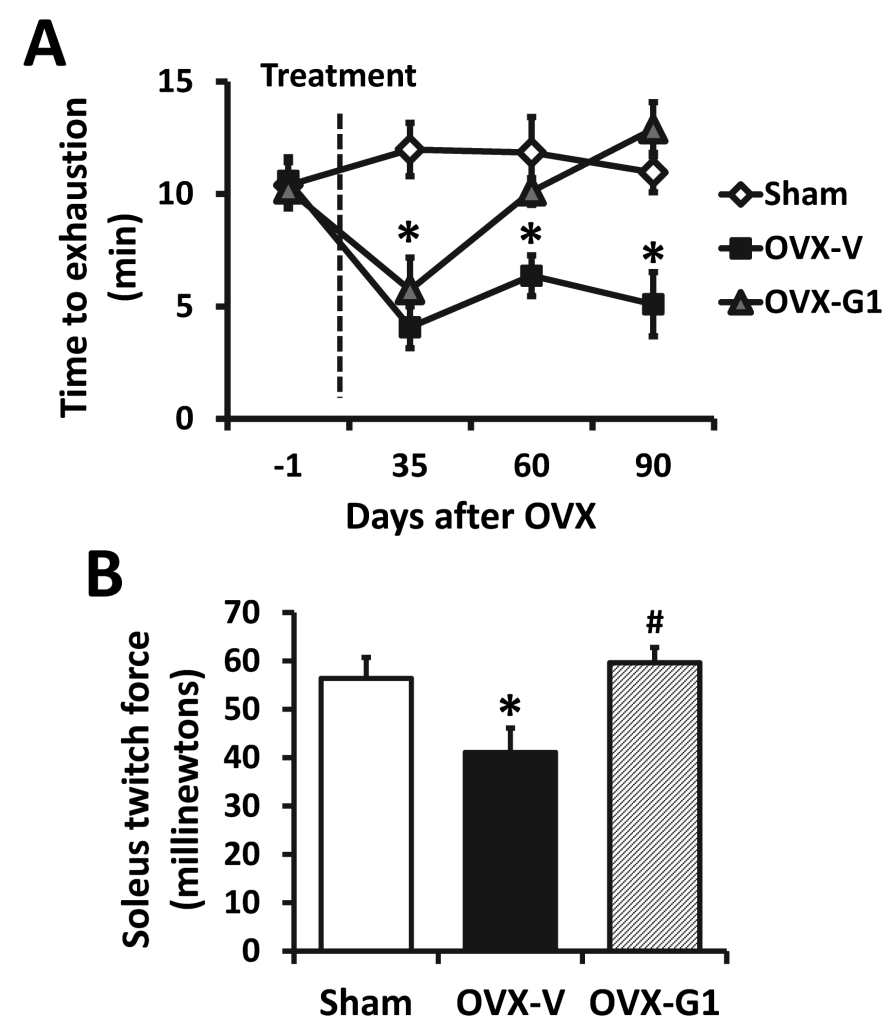

Fig. 2. Time course changes of treadmill performance (A) and peak twitch force of isolated soleus muscle (B) in sham-operated and ovariectomized F344BN rats treated with vehicle or G1 for 2 months

OVX, ovariectomized; V, vehicle. Values are mean $\pm \mathrm{SEM} ;{ }^{*} P<0.05$ vs. sham; $\# P<0.05$ vs. OVX-V. n=7/group. 


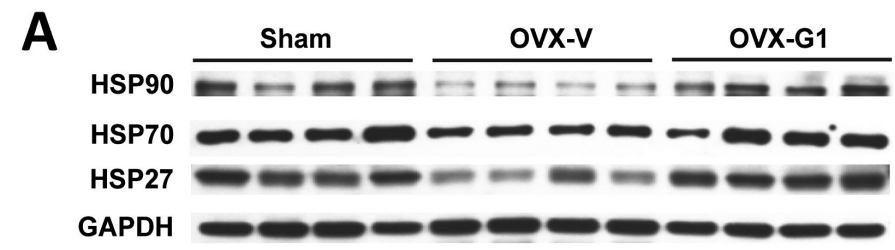

B
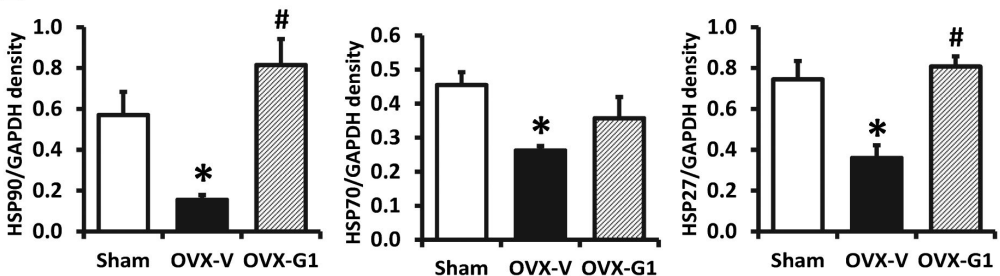

Fig. 3. HSP protein levels in soleus muscles from sham-operated and ovariectomized female F344BN rats treated with vehicle or G1 for 2 months

(A) Representative images of Western blot analysis for HSP90, HSP70, HSP27, and GAPDH as loading control. (B) Quantification of Western blot signal densities using ImageJ. OVX, ovariectomized; V, vehicle. Values are mean \pm SEM; $* P<0.05$ vs. sham; $\# P<0.05$ vs. OVX-V. $\mathrm{n}=7 /$ group. 

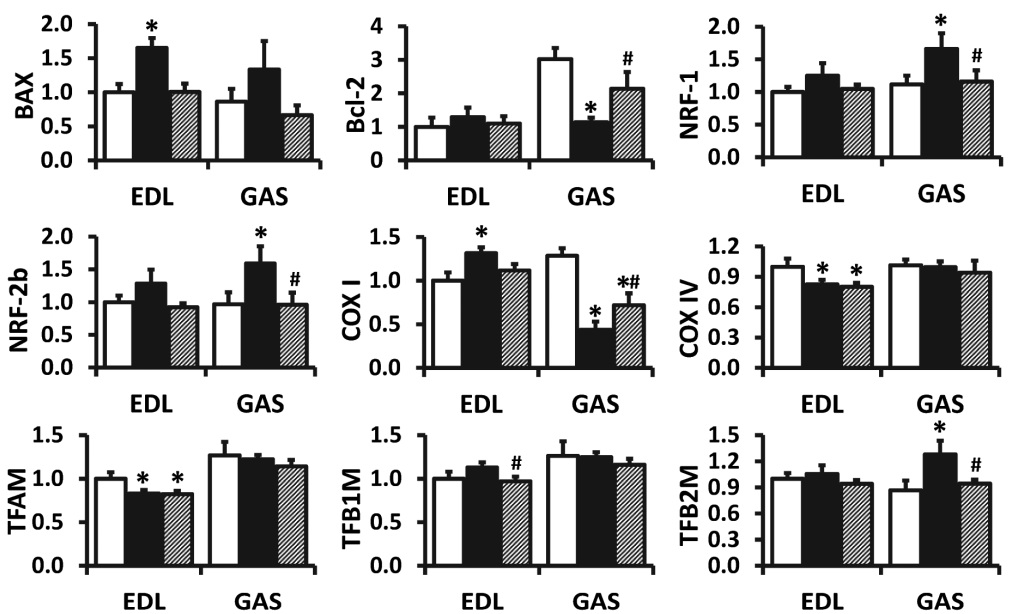

Fig. 4. mRNA levels of mitochondrial and apoptosis-related genes in skeletal muscles of shamoperated and ovariectomized female F344BN rats treated with vehicle or G1 for 2 months Open bar, sham; solid bar, ovariectomized rats treated with vehicle; hatched bar, ovariectomized rats treated with G1; EDL, extensor digitorum longus; GAS, gastrocnemius muscle. Values are mean $\pm \mathrm{SEM} ; * P<0.05$ vs. sham; $\# P<0.05$ vs. OVX-V. $\mathrm{n}=7 /$ group. 